\title{
New top-flavor models with a seesaw mechanism
}

\author{
Hong-Jian He, ${ }^{1}$ Tim M. P. Tait, ${ }^{2}$ and C.-P. Yuan ${ }^{1,3}$ \\ ${ }^{1}$ Michigan State University, East Lansing, Michigan 48824 \\ ${ }^{2}$ Argonne National Laboratory, Argonne, Illinois 60439 \\ ${ }^{3}$ CERN, CH-1211, Geneva, Switzerland \\ (Received 10 November 1999; published 7 June 2000)
}

\begin{abstract}
A new class of models is constructed in which the third family quarks, but not leptons, experience a new $S U(2)$ or $U(1)$ gauge force. Anomaly cancellation enforces the introduction of spectator quarks so that top and bottom quark masses are naturally generated via a seesaw mechanism. We find the new contributions to the $(S, T, U)$ parameters and $Z b \bar{b}$ vertex to be generically small. We analyze how the reasonable flavor mixing pattern can be generated to ensure the top-seesaw mechanism and sufficiently suppress flavor-changing effects for light quarks. Collider signatures for the light Higgs boson and top quark are also discussed.
\end{abstract}

PACS number(s): 12.60.Nz, 11.15.Ex, 12.15.Ff

The single Higgs doublet in the standard model (SM) generates the masses for weak gauge bosons $\left(W^{ \pm}, Z^{0}\right)$ and all quarks and leptons by spontaneous electroweak symmetry breaking (EWSB). However, the striking experimental fact is that only the top quark mass $\left(m_{t}=174.3 \pm 5.1 \mathrm{GeV}\right)$ lies at the same scale as the masses of $\left(W^{ \pm}, Z^{0}\right)$, while all other SM fermions weigh no more than a few GeV. This strongly suggests that the top quark sector may involve certain new gauge dynamics in contrast with all light fermions, including the tau lepton. Following this guideline of model building, we are forced to introduce new spectator fermions associated with the top sector for gauge anomaly cancellation. We then find that the seesaw mechanism is truly generic to the top quark mass generation.

The usual dynamical top-color scenario [1] associates additional strong $S U(3)$ with the top sector, while our topflavor seesaw models involve either extra $S U(2)$ or $U(1)$ and thus predict extra color-singlet heavy gauge bosons such as $W^{\prime}$ and/or $Z^{\prime}$. The old non-universality [2] or top-flavor [4] models assume the entire third family joins the same extra $S U(2)$ gauge group, which fail to explain why the top mass is so much larger than the tau mass while tau is as light as a charm in the second family. The non-commuting extended technicolor (ETC) model [3] has focused on generating a dynamical $m_{t}$ by embedding an extra strong $S U(2)$ into the ETC gauge group with the anomaly issue ignored for simplicity. The recent dynamical top-color seesaw models $[5,6]$ involve an extra singlet heavy quark which is not necessarily required by the anomaly cancellation since the $S U$ (3) top-color can be vector-like for SM quarks and an additional seesaw condition usually needs to be imposed. Our construction stresses that a rigorous realization of topflavor gauge group of either $S U(2)$ or $U(1)$ in the top-sector (but not tau-sector) enforces the introduction of spectator fermions and uniquely leads to a seesaw mechanism for $m_{t}$. The top-flavor with $S U(2)$ gauge group requires spectators only in doublet while our top-flavor $U(1)$ allows either doublet or singlet spectators. The doublet spectator fermions always carry weak isospin so that they more actively participate in the EWSB dynamics than any singlet spectator. The top-flavor seesaw scenario with doublet spectator fermions thus provides a complementary prospect to the original topseesaw idea with an extra singlet quark [5]. As will be shown below, our new top-flavor seesaw models, besides being theoretically well motivated and defined, are fully compatible with low energy data and may further provide exciting collider signatures. An extension to the dynamical symmetry breaking (DSB) scenario is presented.

We construct two types of models in which the top sector, but not tau sector, experiences a new gauge interaction of $S U(2)_{t}$ or $U(1)_{t}$. The full gauge group is $\mathcal{G}_{I}=S U(3)_{c}$ $\otimes S U(2)_{t} \otimes S U(2)_{f} \otimes U(1)_{y}$ (called type-I) or $\mathcal{G}_{I I}=S U(3)_{c}$ $\otimes S U(2)_{w} \otimes U(1)_{t} \otimes U(1)_{f}$ (called type-II). The first two family fermions are singlets under new $S U(2)_{t}$ or $U(1)_{t}$. For the third family, a doublet of spectator quarks $S$ $=(\mathcal{T}, \mathcal{B})^{T}$ is introduced to make the theory free of anomaly (cf. Table I). A complex Higgs scalar $\Phi_{I}=u+\sigma^{0}+i \vec{\tau} \cdot \vec{\chi}$ $\left(\Phi_{I I}=u+\sigma^{0}+i \chi^{0}\right)$, with a nonzero vacuum expectation value $(\mathrm{VEV})$ of $u$, is introduced to break $\mathcal{G}_{I}\left(\mathcal{G}_{I I}\right)$ down to the SM gauge group $\mathcal{G}_{\mathrm{SM}}=S U(3)_{c} \otimes S U(2)_{w} \otimes U(1)_{y}$ at the scale $u(\gg 246 \mathrm{GeV})$, and then a Higgs doublet $H=\left(\pi^{+},(v\right.$ $\left.\left.+h^{0}+i \pi^{0}\right) / \sqrt{2}\right)^{T}$ breaks $\mathcal{G}_{\mathrm{SM}}$ to the electromagnetic $U(1)_{\mathrm{em}}$ at the scale $v \approx 246 \mathrm{GeV}$. (Here, $\vec{\tau}$ is the Pauli matrix.)

The gauge sector of Type-I or -II models contains extra massive color-singlet weak gauge bosons $\left(W^{\prime}, Z^{\prime}\right)$ or $Z^{\prime}$. The basic parameters are a small gauge-mixing angle, $\sin \phi$, between heavy and light gauge bosons, and a large ratio of two VEVs, $x=u^{2} / v^{2} \gg 1$, as often studied in the literature [2-4,7]. In fact, the $Z^{\prime}$ of extra $U(1)$ is generic in grand unified models and string theories [7]. As long as $\sin \phi$ and $1 / x$ are small enough, all the effects of $W^{\prime}$ and/or $Z^{\prime}$ to the low energy processes can be expressed in power expansions of $\sin \phi$ and $1 / x$ [2-4]. For Type-I models, the true VEV $v_{w}$ of the EWSB is related to the VEV $v$ of lighter Higgs boson $h^{0}$ at tree level by $v_{w}=v\left[1-\sin ^{4} \phi / 2 x+O\left(1 / x^{2}\right)\right]$, with the $W$-boson mass $m_{w}=g v_{w} / 2$; while for Type-II models, we have $v_{w}=v$.

The main new feature of our models lies in the Yukawa and Higgs sector, which is the current focus. The scalar $\Phi$ breaks $S U(2)_{t} \otimes S U(2)_{f}\left[U(1)_{t} \otimes U(1)_{f}\right]$ to its diagonal SM group $S U(2)_{w}\left[U(1)_{y}\right]$ in Type-I (II) models at the scale $u(\gg v)$. Consequently, it generates the mass of $W^{\prime}$ and/or $Z^{\prime}$ as well as a physical neutral scalar $\sigma^{0}$. Then, $\mathcal{G}_{\mathrm{SM}}$ breaks down to $U(1)_{\mathrm{em}}$ by the Higgs doublet $H$ at the scale $v$ $\approx 246 \mathrm{GeV}$ and a light neutral Higgs boson $h^{0}$ is generated. 
TABLE I. Quantum number assignments for the third family fermions and the Higgs sector in Type-I and -II models, where $Q_{3 L}=\left(t_{L}, b_{L}\right)^{T}, L_{3}=\left(\nu_{\tau_{L}}, \tau_{L}\right)^{T}$, and $S=(\mathcal{T}, \mathcal{B})^{T}$.

\begin{tabular}{lcccc}
\hline \hline Type-I & $S U(3)_{c}$ & $S U(2)_{t}$ & $S U(2)_{f}$ & $U(1)_{y}$ \\
\hline$Q_{3 L}$ & $\mathbf{3}$ & $\mathbf{2}$ & $\mathbf{1}$ & $1 / 3$ \\
$\left(t_{R}, b_{R}\right)$ & $\mathbf{3}$ & $\mathbf{1}$ & $\mathbf{1}$ & $(4,-2) / 3$ \\
$S_{L}$ & $\mathbf{3}$ & $\mathbf{1}$ & $\mathbf{2}$ & $1 / 3$ \\
$S_{R}$ & $\mathbf{3}$ & $\mathbf{2}$ & $\mathbf{1}$ & $1 / 3$ \\
$L_{3}$ & $\mathbf{1}$ & $\mathbf{1}$ & $\mathbf{2}$ & -1 \\
$\tau_{R}$ & $\mathbf{1}$ & $\mathbf{1}$ & $\mathbf{1}$ & -2 \\
\hline$\Phi$ & $\mathbf{1}$ & $\mathbf{2}$ & $\mathbf{2}$ & 0 \\
$H$ & $\mathbf{1}$ & $\mathbf{1}$ & $\mathbf{2}$ & 1 \\
\hline Type-II & $S U(3)_{c}$ & $S U(2)_{w}$ & $U(1)_{t}$ & $U(1)_{f}$ \\
\hline$Q_{3 L}$ & $\mathbf{3}$ & $\mathbf{2}$ & $1 / 3$ & 0 \\
$\left(t_{R}, b_{R}\right)$ & $\mathbf{3}$ & $\mathbf{1}$ & 0 & $(4,-2) / 3$ \\
$S_{L}$ & $\mathbf{3}$ & $\mathbf{2}$ & 0 & $1 / 3$ \\
$S_{R}$ & $\mathbf{3}$ & $\mathbf{2}$ & $1 / 3$ & 0 \\
$L_{3}$ & $\mathbf{1}$ & $\mathbf{2}$ & 0 & -1 \\
$\tau_{R}$ & $\mathbf{1}$ & $\mathbf{1}$ & 0 & -2 \\
\hline$\Phi$ & $\mathbf{1}$ & $\mathbf{1}$ & $-1 / 3$ & $1 / 3$ \\
$H$ & $\mathbf{1}$ & $\mathbf{2}$ & 0 & 1 \\
\hline \hline
\end{tabular}

Therefore, in contrast to the usual two Higgs doublet model (2HDM), our models have no charged Higgs bosons. There are a pair of neutral scalars $\left(h^{0}, \sigma^{0}\right)$ with a mixing angle $\alpha$. The value of $\alpha$ depends on the details of the scalar potential $V\left(h^{0}, \sigma^{0}\right)$ and will be treated as a free parameter below. As a result of the spontaneous symmetry breaking, the scalars are expected to obtain tree level masses of the order of their VEV's, i.e., $m_{h} \sim v \sim O(100 \mathrm{GeV})$ and $M_{\sigma} \sim u \sim O(\mathrm{TeV})$.

Defining $\widetilde{H}=-i \tau^{2} H^{*}$, from Table I, we find the following Yukawa interactions of the third family quarks for both Type-I and -II:

$-\frac{y_{s}}{\sqrt{2}} \overline{S_{L}} \Phi S_{R}-y_{s t} \overline{S_{L}} \widetilde{H} t_{R}-y_{s b} \overline{S_{L}} H b_{R}-\kappa \overline{Q_{L}^{3}} S_{R}+$ H.c.,

which generate top- and bottom-seesaw mass matrices:

$$
\begin{aligned}
& -\left(\overline{t_{L}}, \overline{\mathcal{T}_{L}}\right)\left(\begin{array}{cc}
0 & \kappa \\
m_{s t} & M_{S}
\end{array}\right)\left(\begin{array}{l}
t_{R} \\
\mathcal{T}_{R}
\end{array}\right) \\
& -\left(\overline{b_{L}}, \overline{\mathcal{B}_{L}}\right)\left(\begin{array}{cc}
0 & \kappa \\
m_{s b} & M_{S}
\end{array}\right)\left(\begin{array}{l}
b_{R} \\
\mathcal{B}_{R}
\end{array}\right)+\text { H.c., }
\end{aligned}
$$

where $M_{S}=y_{s} u / \sqrt{2}, m_{s t}=y_{s t} v / \sqrt{2}, m_{s b}=y_{s b} v / \sqrt{2}$. The parameter $\kappa$, allowed before spontaneous symmetry breaking, is expected to be of $O\left(M_{S}\right)$. Because of the doublet nature of $(\mathcal{T}, \mathcal{B})$ in our model, the same $\kappa$ appears in both top and bottom seesaw, in contrast to the recent dynamical seesaw models with singlet $\chi$ and $\omega$ quarks $[5,6]$. For the parameter space $M_{S} \gtrsim \kappa \gg m_{s t}>m_{t}$, the mass eigenvalues of $(t, b)$ and $(\mathcal{T}, \mathcal{B})$ can be expanded as

$$
\begin{aligned}
& m_{t}=\frac{m_{s t} \kappa}{M_{S} \sqrt{1+r}}\left[1-\frac{\left(m_{s t} / M_{S}\right)^{2}}{2(1+r)^{2}}+O\left(\frac{m_{t}^{4}}{M_{S}^{4}}\right)\right], \\
& m_{b}=\frac{m_{s b} \kappa}{M_{S} \sqrt{1+r}}\left[1-\frac{\left(m_{s b} / M_{S}\right)^{2}}{2(1+r)^{2}}+O\left(\frac{m_{b}^{4}}{M_{S}^{4}}\right)\right], \\
& M_{\mathcal{T}}=M_{S} \sqrt{1+r}\left[1+\frac{z_{t}^{2}}{2(1+r)}+\frac{4 r+3}{8(1+r)^{2}} z_{t}^{4}+O\left(z_{t}^{6}\right)\right], \\
& M_{\mathcal{B}}=M_{S} \sqrt{1+r}\left[1+\frac{z_{b}^{2}}{2(1+r)}+\frac{4 r+3}{8(1+r)^{2}} z_{b}^{4}+O\left(z_{b}^{6}\right)\right],
\end{aligned}
$$

where $r \equiv\left(\kappa / M_{S}\right)^{2} \sim O(1)$ and $z_{t(b)} \equiv m_{t(b)} / \kappa$ with $z_{b} \ll z_{t}$ $\ll 1$. The mass splitting of the heavy quarks $(\mathcal{T}, \mathcal{B}), \Delta M_{\mathcal{T B}}$ $=M_{\mathcal{T}}-M_{\mathcal{B}}$, is thus deduced as

$$
\Delta M_{\mathcal{T B}}=m_{t}\left\{z_{t}[2 \sqrt{r(1+r)}]^{-1}+O\left(z_{t}^{3}\right)\right\} \ll m_{t} .
$$

The tiny mass splitting of the $(\mathcal{T}, \mathcal{B})$ doublet is essential for satisfying the high precision bound of the $\delta \rho$ or $T$ parameter [8].

The seesaw mass matrices in (2) are diagonalized by 2 $\times 2$ bi-unitary transformations, $K_{L}^{j \dagger} \mathcal{M}^{j} K_{R}^{j}=\mathcal{M}_{\text {diag }}^{j}$, where the superscript $j \in(t, b)$ specifies the up- and down-type rotations. The rotation angles $\left(\theta_{L}^{j}, \theta_{R}^{j}\right)$ are

$$
\begin{aligned}
& \sin \theta_{R}^{j}=\frac{z_{j}}{\sqrt{1+r}}\left[1+\frac{r}{1+r} z_{j}^{2}\right]+O\left(z_{j}^{5}\right), \\
& \sin \theta_{L}^{j}=\sqrt{\frac{r}{1+r}}\left[1-\frac{z_{j}^{2}}{1+r}-\frac{3 r z_{j}^{4}}{2(1+r)^{2}}+O\left(z_{j}^{6}\right)\right] .
\end{aligned}
$$

Since $z_{b} / z_{t}=m_{b} / m_{t} \sim 1 / 40 \ll 1$, the seesaw rotation effects from the bottom sector are much smaller than that in the top sector. If we consider the typical situation with $z_{b} \lesssim O\left(z_{t}^{2}\right)$, the tiny contribution from the bottom rotations to $(S, T, U)$ and $R_{b}$ can be ignored.

With the above seesaw rotations and the $\alpha$ rotation of $\left(h^{0}, \sigma^{0}\right)$ from the Higgs potential, we derive from (1) the following Yukawa interactions of $\left(h^{0}, \sigma^{0}\right)$ with $t \bar{t}$ and $b \bar{b}$ in the unitary gauge, up to $O\left(z_{t}^{2}, z_{b}\right)$,

$$
\begin{aligned}
& -\frac{m_{t}}{v}\left[c_{\alpha}\left(1-\frac{z_{t}^{2}}{1+r}\right)-s_{\alpha} \frac{x^{-1 / 2}}{1+r}\left(1-\frac{1-r}{1+r} z_{t}^{2}\right)\right] h^{0} \bar{t} t \\
& \quad+\frac{m_{t}}{v}\left[s_{\alpha}\left(1-\frac{z_{t}^{2}}{1+r}\right)+c_{\alpha} \frac{x^{-1 / 2}}{1+r}\left(1-\frac{1-r}{1+r} z_{t}^{2}\right)\right] \sigma^{0} \bar{t} t \\
& \quad-\frac{m_{b}}{v}\left[c_{\alpha}-s_{\alpha} \frac{x^{-1 / 2}}{1+r}\right] h^{0} \bar{b} b+\frac{m_{b}}{v}\left[s_{\alpha}+c_{\alpha} \frac{x^{-1 / 2}}{1+r}\right] \sigma^{0} \bar{b} b
\end{aligned}
$$

where $-\pi / 2 \leqslant \alpha \leqslant 0$ and $\left(s_{\alpha}, c_{\alpha}\right) \equiv(\sin \alpha, \cos \alpha)$. Thus, the $h t \bar{t}$ coupling may be significantly different from the SM value of $m_{t} / v_{w}$ depending on the parameter space of $\left(\alpha, x, M_{S}, \kappa\right)$. This may provide, for instance, important 
non-SM signatures via the processes $g g \rightarrow h^{0}\left(\rightarrow W^{*} W^{*}\right.$ $\rightarrow l \nu l \nu)$ at the Tevatron, $g g \rightarrow h^{0}, \quad g g \rightarrow \sigma^{0} \rightarrow h^{0} h^{0}$ and $W W \rightarrow t \bar{t}$ at the CERN Large Hadron Collider (LHC), and $e^{-} e^{+} \rightarrow h^{0} t \bar{t}, \nu \bar{\nu} t \bar{t}$ at the high energy linear colliders.

The three main new contributions to $(S, T, U)$ and $Z b \bar{b}$ arise from (a) the small mixings of heavy $W^{\prime}$ and/or $Z^{\prime}$ with $W(Z)$; (b) the $\mathcal{T}$ - $t$ and $\mathcal{B}-b$ mixings from the seesaw mechanism as well as the $(\mathcal{T}, \mathcal{B})$ doublet itself; (c) the mixing of the Higgs bosons. The type-(a) contribution is generic to any extended gauge sector with a breaking pattern $S U(2)_{1}$ $\otimes S U(2)_{2} \rightarrow S U(2)_{w}$ or $U(1)_{1} \otimes U(1)_{2} \rightarrow U(1)_{y}$ and can safely fit the data as long as the mixing angle $\sin \phi$ and the ratio $1 / x$ are small enough $[4,7]$. Our real concern is the new type-(b) and -(c) corrections. The usual expectation is that only $S U(2)_{w}$ singlet heavy fermions are phenomenologically safe [5], but our analysis shows that the contributions of the doublet fermions $(\mathcal{T}, \mathcal{B})$ in our seesaw mechanism are also generically small enough to agree with the current data. For simplicity, we compute the type-(b) contributions up to $O\left(z_{t}^{4}\right)$ in seesaw expansion while keeping leading orders in small $\sin \phi$ and $1 / x$ expansions $[4,7]$. To the leading order in $\sin \phi$ and $1 / x$, the doublet $(\mathcal{T}, \mathcal{B})$ behaves essentially vectorlike under the SM gauge group, and thus their heavy masses are expected to respect the decoupling theorem [9]. Even though the masses $M_{S}$ and $\kappa$ are invariant under $\mathcal{G}_{\mathrm{SM}}$, the other seesaw mass terms $\left(m_{s t}\right.$ and $\left.m_{s b}\right)$ are not. It is a nontrivial task to confirm that the spectator-fermion corrections to $(S, T, U)$ can decouple sufficiently since the fermion loops involving heavy $\mathcal{T} / \mathcal{B}$ do contribute dangerous $O\left(M_{S}^{2}\right)$ and $O\left(M_{S}^{0}\right)$ terms to the self-energies of $W / Z$.

The calculations of $(S, T, U)$ are tedious, but the results to $O\left(z_{t}^{2}, z_{b}\right)$ can be compactly summarized,

$$
\begin{aligned}
& S=\frac{4 N_{c}}{9 \pi}\left[\ln \frac{M_{\mathcal{T}}}{m_{t}}-\frac{7}{8}+\frac{1}{16 h_{t}}-\frac{1}{560 h_{t}^{2}}\right] \frac{z_{t}^{2}}{1+r}, \\
& T=\frac{N_{c} h_{t}}{16 \pi s_{w}^{2} c_{w}^{2}}\left[8 \ln \frac{M_{\mathcal{B}}}{m_{t}}+\frac{4}{3 r}-6\right] \frac{z_{t}^{2}}{1+r}, \\
& U=\frac{N_{c}}{6 \pi}\left[1+\frac{1}{10 h_{t}}+\frac{1}{70 h_{t}^{2}}\right] \frac{z_{t}^{2}}{1+r},
\end{aligned}
$$

where $h_{t} \equiv\left(m_{t} / m_{z}\right)^{2}$ and we have ignored tiny $O\left(1 / h_{t}^{3}\right)$ terms inside $[\cdots]$. $\left(s_{w} \equiv \sin \theta_{W}\right.$ and $\theta_{W}$ is the weak mixing angle.) We see that these new contributions are phenomenologically safe since $z_{t}^{2} \ll 1$. For instance, taking $M_{S}=2 \kappa$ $=5 \mathrm{TeV}$, we have $(S, T, U)=\left(4 \times 10^{-3}, 0.13,6 \times 10^{-4}\right)$, while choosing $\left(M_{S}, \kappa\right)=(5,4) \mathrm{TeV}$, we get $(S, T, U)$ $=\left(1.4 \times 10^{-3}, 0.04,2 \times 10^{-4}\right)$. So, the seesaw corrections to $(S, U)$ are generally negligible.

There are also contributions to $(S, T, U)$ from the Higgs bosons. In the limit of $1 / x \ll 1$, the heavy $\sigma^{0}$ only indirectly couples to $W / Z$ via its $\alpha$ mixing with the light $h^{0}$. The interactions of $\left(h^{0}, \sigma^{0}\right)$ with $\left(W^{ \pm}, Z^{0}\right)$ are

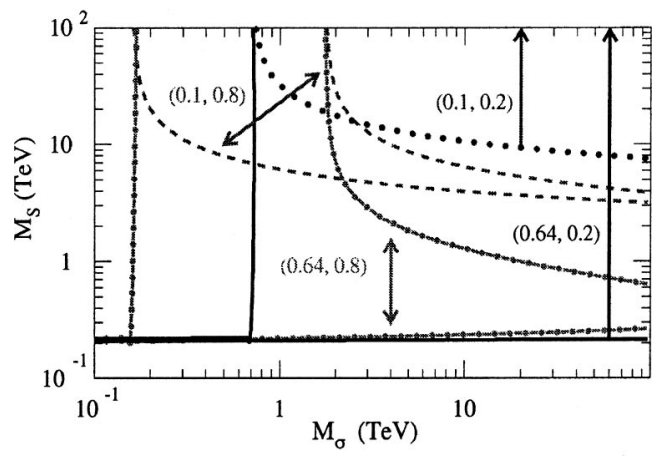

FIG. 1. Constraints on $\left(M_{\sigma}, M_{S}\right)$ by $(S, T)$ fit at $95 \%$ C.L., for $m_{h}=100 \mathrm{GeV}$ and four sets of $\left(r, s_{\alpha}^{2}\right)$ as shown. The allowed regions (indicated by arrows) lie between two lines (or above one line) appropriate to each parameter set.

$$
\begin{aligned}
\frac{e^{2}}{2 s_{w}^{2}} & W_{\mu}^{2}\left(c_{\alpha} h^{0}+s_{\alpha} \sigma^{0}\right)^{2}+\frac{e^{2}}{2 s_{w}^{2} c_{w}^{2}} Z_{\mu}^{2}\left(c_{\alpha} h^{0}+s_{\alpha} \sigma^{0}\right)^{2} \\
& +\frac{e m_{w}}{s_{w}} W_{\mu}^{2}\left(c_{\alpha} h^{0}+s_{\alpha} \sigma^{0}\right)+\frac{e m_{z}}{s_{w} c_{w}} Z_{\mu}^{2}\left(c_{\alpha} h^{0}+s_{\alpha} \sigma^{0}\right) .
\end{aligned}
$$

Thus, the $\left(h^{0}, \sigma^{0}\right)$ contribute to low energy observables in the same way as the SM Higgs boson, but with a scaling factor of $\left(c_{\alpha}^{2}, s_{\alpha}^{2}\right)$. From the SM Higgs correction to $(S, T, U)$ [8], we derive the additional contributions from $\left(h^{0}, \sigma^{0}\right)$ :

$$
\begin{aligned}
& \Delta S=\frac{1}{12 \pi}\left[c_{\alpha}^{2} \ln \frac{m_{h}^{2}}{m_{z}^{2}}-\ln \frac{\left(m_{h}^{2}\right)_{\mathrm{ref}}^{\mathrm{sm}}}{m_{z}^{2}}+s_{\alpha}^{2} \ln \frac{M_{\sigma}^{2}}{m_{z}^{2}}\right], \\
& \Delta T=\frac{-3}{16 \pi c_{w}^{2}}\left[c_{\alpha}^{2} \ln \frac{m_{h}^{2}}{m_{z}^{2}}-\ln \frac{\left(m_{h}^{2}\right)_{\mathrm{ref}}^{\mathrm{sm}}}{m_{z}^{2}}+s_{\alpha}^{2} \ln \frac{M_{\sigma}^{2}}{m_{z}^{2}}\right],
\end{aligned}
$$

and $\Delta U \simeq 0$, where $\left(m_{h}\right)_{\mathrm{ref}}^{\mathrm{sm}}$ is the reference value of the SM Higgs mass. For $\left(m_{h}\right)_{\mathrm{ref}}^{\mathrm{sm}}=m_{h}=100 \mathrm{GeV}$ and $M_{\sigma}=1 \mathrm{TeV}$, we find $(\Delta S, \Delta T)=(0.02,-0.07)$ with $s_{\alpha}^{2}=0.2$. The contributions of the Higgs and seesaw sectors to $T$ can have opposite signs, which makes our model easily accommodate the data with a small $T$ for reasonable $\left(M_{\sigma}, M_{S}\right)$. On the other hand, $M_{S}$ is bounded from above (since a larger $M_{S}$ lifts up $S$ to the positive side) and also from below (since a light $M_{S}$ pushes both $S$ and $T$ towards negative). Considering $1 / x \ll 1$ and summing up dominant contributions in the Higgs and seesaw sectors, we can derive constraints in the $\left(M_{\sigma}, M_{S}\right)$ plane from the precision fit of $(S, T)$ [10], as shown in Fig. 1. We have chosen $\left(m_{h}\right)_{\text {ref }}^{\mathrm{sm}}=100 \mathrm{GeV}$ for the $(S, T)$ fit, with the complete 1-loop SM corrections included (in accord with the precision of our 1-loop new physics results). The fitted values of $(S, T)=(0.13 \pm 0.11,-0.13 \pm 0.14)$ deviate from $(0,0)$ at $1 \sigma$ level. Figure 1 shows that $M_{\sigma}$ is always bounded from below since a too light $\sigma^{0}$ drives both $(\Delta S, \Delta T)$ to zero.

We finally discuss the ratio of $Z$ decay width $R_{b}=\Gamma(Z$ $\rightarrow b \bar{b}) / \Gamma(Z \rightarrow$ hadrons $)$ and the $Z b \bar{b}$ coupling asymmetry $A_{b}=\left(g_{b L}^{2}-g_{b R}^{2}\right) /\left(g_{b L}^{2}+g_{b R}^{2}\right)$. The current experimental data from $R_{b}$ and $A_{b}$ can be translated into the bounds on the allowed deviation of the $Z b \bar{b}$ couplings $\left(g_{b L}, g_{b R}\right)$ from 
their SM values, $0.002 \leqslant \delta g_{b L} \leqslant 0.009$ and $0.004 \leqslant \delta g_{b R}$ $\leqslant 0.036$, at the $2 \sigma$ level [11]. It is straightforward to compute the corrections to $Z b \bar{b}$ couplings from the seesaw sector of our model. The correction associated with the top sector only comes from loop and is of $O\left(z_{t}^{2}\right)$ so that it is generally small, but the bottom seesaw induces a tree level correction $\delta g_{b R}^{\text {new }}$ to the right-handed $Z b \bar{b}$ coupling,

$$
\delta g_{b R}^{\text {new }}=-e /\left(2 s_{w} c_{w}\right)\left(\sin \theta_{R}^{b}\right)^{2} .
$$

This negative correction is at the order of $\left(\sin \theta_{R}^{b}\right)^{2}$ $\simeq\left(m_{b} / \kappa\right)^{2} /(1+r) \leq O\left(10^{-6}-10^{-8}\right)$ for $\quad \kappa=O(1-10) \mathrm{TeV}$ and thus is essentially negligible. This feature is different from the recent dynamical seesaw models with singlets $\chi$ and $\omega$ in which the left-handed (instead of right-handed) $b-\omega$ mixings contribute to the $Z b \bar{b}$ vertex [6]. Another nice feature is that our models contain no charged Higgs boson and are thus free of its undesirable negative correction to $R_{b}$ and also its enhancement to $b \rightarrow s \gamma$ decay rate in the usual 2HDM [11].

To establish realistic flavor mixings among all three families with the well constrained Cabibbo-Kobayashi-Maskawa (CKM) matrix generated is a more challenging task. We do not want to spoil the seesaw pattern of the mass matrices in (2) after the mixings with the first two family fermions are included, and we also need to properly suppress flavorchanging effects associated with the light quarks. The quantum number assignments in Table I do not automatically suppress the mixings of $(\mathcal{T}, \mathcal{B})$ and $(t, b)$ with light fermions. We impose a simple discrete $Z_{4}$ symmetry to ensure the desired pattern of the $4 \times 4$ mass matrices for up- and downtype quarks. Under $Z_{4}=\exp ($ in $\pi / 2)$ with $n \in(0,1,2,3)$, we define the following field transformations:

$$
\begin{gathered}
Q_{3 L} \rightarrow i Q_{3 L}, \quad S_{R} \rightarrow i S_{R}, \quad \Phi \rightarrow i \Phi, \\
\left(t_{R}, b_{R}\right) \rightarrow-\left(t_{R}, b_{R}\right), \quad S_{L} \rightarrow-S_{L},
\end{gathered}
$$

and other fields are unchanged by $Z_{4}$. Then, we can write down all relevant effective operators in the quark Yukawa sector, invariant under $\mathcal{G}_{I}\left(\mathcal{G}_{I I}\right)$. For instance, in the Type-I models, the Yukawa Lagrangian $-\mathcal{L}_{Y(U)}$ of the up-type quarks becomes

$$
\begin{gathered}
\sum_{i, j=1}^{2} y_{i j} \overline{Q_{i L}} \widetilde{H} u_{j R}+y_{i 3} \overline{Q_{i L}} \widetilde{H} t_{R} \frac{\operatorname{det} \Phi}{\Lambda_{f}^{2}}+\frac{y_{i 4}}{\sqrt{2}} \overline{Q_{i L}} \Phi S_{R} \frac{\operatorname{det} \Phi}{\Lambda_{f}^{2}} \\
+y_{3 j} \overline{Q_{3 L}} \Phi^{\dagger} \widetilde{H} u_{j R} \frac{\operatorname{det} \Phi}{\Lambda_{f}^{3}}+y_{33} \overline{Q_{3 L}} \frac{\Phi^{\dagger}}{\Lambda_{f}} \widetilde{H} t_{R}+\kappa \overline{Q_{3 L}} S_{R} \\
+y_{4 j} \overline{S_{L}} \widetilde{H} u_{j R} \frac{\operatorname{det} \Phi}{\Lambda_{f}^{2}}+y_{t s} \overline{S_{L}} \widetilde{H} t_{R}+\frac{y_{S}}{\sqrt{2}} \overline{S_{L}} \Phi S_{R}
\end{gathered}
$$

where $\Lambda_{f}$ is the cutoff scale of the flavor symmetry breaking. Defining $m_{i j}=y_{i j} v / \sqrt{2}$, we find that the resulting mass matrix for $(u, c, t, \mathcal{T})$ poses a natural hierarchy,

$$
\mathcal{M}_{u}=\left(\begin{array}{cccc}
m_{11} & m_{12} & m_{13} \epsilon^{2} & m_{14} \delta \\
m_{21} & m_{22} & m_{23} \epsilon^{2} & m_{24} \delta \\
m_{31} \epsilon^{3} & m_{33} \epsilon^{3} & m_{33} \epsilon & \kappa \\
m_{41} \epsilon^{2} & m_{42} \epsilon^{2} & m_{s t} & M_{S}
\end{array}\right),
$$

in which $\epsilon=u / \Lambda_{f}$ and $\delta=\epsilon^{2}(u / v)$ are small parameters. The down-type quarks exhibit a similar pattern in $\mathcal{M}_{d}$. A proper bi-unitary field transformation, containing the dominating 2 $\times 2$ seesaw rotations in the $t-\mathcal{T}(b-\mathcal{B})$ sector, can first rotate away the small mixings of $\mathcal{T}(\mathcal{B})$ with all light quarks so that the $4 \times 4$ mass matrix reduces to $3 \times 3$ for the three-family quarks of the SM, i.e.,

$$
\hat{\mathcal{M}}_{u}=\left(\begin{array}{ccc}
m_{11} & m_{12} & m_{13}^{\prime} \delta \\
m_{21} & m_{22} & m_{23}^{\prime} \delta \\
m_{31}^{\prime} \epsilon^{2} & m_{32}^{\prime} \epsilon^{2} & m_{t}^{\prime}
\end{array}\right),
$$

where $m_{t}^{\prime} \leqslant m_{t}$. A similar analysis applies to Type-II models. Following the procedure of Ref. [12], realistic CKM mixings of SM fermions can be generated with a proper construction of left-handed rotations for the up- and down-type quarks. The flavor changing effects associated with light quarks were found to be reasonably suppressed [12] in consistency with low energy data, while right-handed mixings are constrained by the mass pattern (14). Sizable flavor mixings between right-handed $c_{R}$ and $t_{R}$ are allowed [12]:

$$
K_{U R}^{t c} \lesssim \sqrt{1-\left(m_{t}^{\prime} / m_{t}\right)^{2}} \simeq 0.11-0.33
$$

for reasonable values of $\delta m_{t}=m_{t}-m_{t}^{\prime}=O(1-10) \mathrm{GeV}$. Hence, the charm-gluon fusion process $g c \rightarrow h^{0} t$ [13] provides an important Higgs boson discovery channel at the LHC.

While the above top-flavor seesaw models have provided the crucial ingredients on how a large top mass is generated together with the EWSB, it is desirable to invoke dynamical symmetry breaking at the $\mathrm{TeV}$ scale without introducing fundamental Higgs bosons. Here, we only consider the simplest DSB realization of our seesaw mechanism of Type-II models, which is called Type-IID below.

To replace the fundamental VEV $\langle H\rangle$ by a dynamical condensate, we may introduce a strong $S U(3)_{t}$ gauge interaction for $\left(t_{R}, b_{R}\right)$ and $\mathcal{S}_{L}$ while all other quarks join the weaker $S U(3)_{f}$ group. [The strong $S U(3)_{t}$ is traditionally called top-color [1].] Thus, our Type-IID models, as an extension of the above Type-II scenario, have the gauge structure $\quad \mathcal{G}_{I I D}=S U(3)_{t} \otimes S U(3)_{f} \otimes S U(2)_{w} \otimes U(1)_{t} \otimes U(1)_{f}$, which turns out to match the gauge group of the original non-seesaw top-color models [1]. But our Type-IID models differ in that they contain new doublet spectator fermions for generating the seesaw mechanism and have very different quantum number arrangement enforced by the anomaly cancellation (cf. Table II). The first two family fermions are charged under weaker $S U(3)_{f}$ and $U(1)_{f}$ as in the SM. The strong $U(1)_{t}$ is now designed to tilt the vacuum such that only top but not bottom gets a large seesaw mass, cf. (17),(18). 
TABLE II. Quantum number assignments for the third family fermions and the effective Higgs scalar $\Phi$ in Type-IID models.

\begin{tabular}{lccccr}
\hline \hline Type-IID & $S U(3)_{t}$ & $S U(3)_{f}$ & $S U(2)_{w}$ & $U(1)_{t}$ & $U(1)_{f}$ \\
\hline$Q_{3 L}$ & $\mathbf{1}$ & $\mathbf{3}$ & $\mathbf{2}$ & 0 & $1 / 3$ \\
$\left(t_{R}, b_{R}\right)$ & $\mathbf{3}$ & $\mathbf{1}$ & $\mathbf{1}$ & $(4,-2) / 3$ & 0 \\
$S_{L}$ & $\mathbf{3}$ & $\mathbf{1}$ & $\mathbf{2}$ & $1 / 3$ & 0 \\
$S_{R}$ & $\mathbf{1}$ & $\mathbf{3}$ & $\mathbf{2}$ & 0 & $1 / 3$ \\
$L_{3}$ & $\mathbf{1}$ & $\mathbf{1}$ & $\mathbf{2}$ & -1 & 0 \\
$\tau_{R}$ & $\mathbf{1}$ & $\mathbf{1}$ & $\mathbf{1}$ & -2 & 0 \\
\hline$\Phi$ & $\mathbf{3}$ & $\overline{\mathbf{3}}$ & $\mathbf{1}$ & $1 / 3$ & $-1 / 3$ \\
\hline \hline
\end{tabular}

The gauge group $\mathcal{G}_{I I D}$ first breaks down to $\mathcal{G}_{\mathrm{SM}}$ at the scale $u$ and then breaks down to $U(1)_{\mathrm{em}}$ at the scale $v$. The first step breaking may be effectively parametrized by a scalar $\Phi$ with VEV $u$, from which the massive octet colorons $\left(G_{\mu}^{\prime a}\right)$ and $U(1)$ gauge boson $\left(Z_{\mu}^{\prime}\right)$ are generated at the scale $M_{c} \sim M_{y} \lesssim 4 \pi u$. $\left(M_{c}\right.$ and $M_{y}$ are the masses of $G_{\mu}^{\prime a}$ and $Z_{\mu}^{\prime}$, respectively.) Thus, integrating out the heavy $G_{\mu}^{\prime a}$ and $Z_{\mu}^{\prime}$ results in the effective interaction for the third family quarks:

$$
\begin{aligned}
& -\frac{4 \pi \kappa_{c}}{M_{c}^{2}}\left(\overline{S_{L}} \gamma^{\mu} \frac{\lambda^{a}}{2} S_{L}+\overline{t_{R}} \gamma^{\mu} \frac{\lambda^{a}}{2} t_{R}+\overline{b_{R}} \gamma^{\mu} \frac{\lambda^{a}}{2} b_{R}\right)^{2} \\
& -\frac{4 \pi \kappa_{y}}{M_{y}^{2}}\left(\frac{1}{6} \overline{S_{L}} \gamma^{\mu} S_{L}+\frac{2}{3} \overline{t_{R}} \gamma^{\mu} t_{R}-\frac{1}{3} \overline{b_{R}} \gamma^{\mu} b_{R}\right)^{2} .
\end{aligned}
$$

Here, $\left(\kappa_{c}, \kappa_{y}\right)=\left(g_{3}^{2} \cot ^{2} \theta, g^{\prime 2} \cot ^{2} \theta^{\prime}\right) / 8 \pi$, with $g_{3}\left(g^{\prime}\right)$ the gauge coupling of the SM color (hypercharge) force and $\theta\left(\theta^{\prime}\right)$ the mixing angle of the two $S U(3)$ 's $[U(1)$ 's] [1]. Applying the Fierz transformation to (16) leads to NambuJona-Lasinio (NJL) type interactions, for $M_{y} \simeq M_{c}$,

$\frac{8 \pi}{M_{c}^{2}}\left[\left(\bar{\kappa}_{c}+\frac{2 \kappa_{y}}{9 N_{c}}\right)\left({\overline{S_{L}}}_{R}\right)\left(\overline{t_{R}} S_{L}\right)+\left(\bar{\kappa}_{c}-\frac{\kappa_{y}}{9 N_{c}}\right)\left(\overline{S_{L}} b_{R}\right)\left(\overline{b_{R}} S_{L}\right)\right]$,

where $\bar{\kappa}_{c}=\kappa_{c}\left(1-1 / N_{c}^{2}\right)$. In the large- $N_{c}$ expansion, a generic NJL-type vertex, $\hat{\kappa} \Lambda^{-2}\left(\overline{X_{L}} Y_{R}\right)\left(\overline{Y_{R}} X_{L}\right)$, has a critical coupling $\hat{\kappa}_{\text {crit }} \simeq 8 \pi^{2} / N_{c}$ for the dynamical condensation. With the $U(1)_{t}$ tilting in (17), we thus have $\left\langle\overline{\mathcal{T}_{L}} t_{R}\right\rangle \neq 0$ and $\left\langle\overline{\mathcal{B}_{L}} b_{R}\right\rangle=0$, provided

$$
3 \pi / 8-\kappa_{y} / 12 \leqslant \kappa_{c} \leqslant 3 \pi / 8+\kappa_{y} / 24
$$

An essential feature of our scenario is that the spectator $\mathcal{T}_{L}$, but not the SM $t_{L}$, plays the key role in the dynamical condensate which generates the EWSB and seesaw top mass, in contrast to the recent top-seesaw models involving extra singlet heavy quark $[5,6]$. Consequently, two composite Higgs doublets $H_{s t}$ and $H_{s b}$ are generated, which are made of $\left(\overline{S_{L}} t_{R}\right)$ and $\left(\overline{S_{L}} b_{R}\right)$, respectively. The $U(1)$ tilting in (18) ensures that $\left\langle H_{s t}\right\rangle \neq 0$ and $\left\langle H_{s b}\right\rangle=0$. Thus, the Higgs spectrum contains a top-condensate Higgs boson $h_{s t}^{0}$, a $b$-Higgs boson $h_{s b}^{0}$, and three $b$ pions $\left(\pi_{s b}^{0}, \pi_{s b}^{ \pm}\right)$, as hybirds between $\left(\mathcal{T}_{L}, \mathcal{B}_{L}\right)$ and $\left(t_{R}, b_{R}\right)$. With the coloron mass $M_{c} \lesssim 4 \pi u$ as a cutoff, we can now re-derive the Pagels-Stokar formula for generating both the dynamical top mass and EWSB with $v$ $\approx 246 \mathrm{GeV}$, i.e.,

$$
v^{2}=\frac{N_{c}}{8 \pi^{2}} \frac{m_{t}^{2}}{\sin ^{2} \theta_{L}^{t}} \ln \frac{M_{c}^{2}}{M_{S}^{2}(1+r)}+O\left(z_{t}^{2}\right)
$$

where, for example, $\left(\kappa, M_{S}, M_{c}\right) \sim(2,5,50) \mathrm{TeV}$ and $m_{t} / \sin \theta_{L}^{t} \sim 600 \mathrm{GeV}$. Note that Eq. (19) involves the lefthanded (instead of right-handed) seesaw rotation angle $\theta_{L}^{t}$, unlike the situation in Refs. [5,6].

As a final remark, the small masses of $b, \tau$, and the first two family fermions have to be generated by different mechanisms, which are much more model-dependent [14]. For instance, they can come from higher dimensional effective operators [6], composite Higgs doublet (formed at higher scale) with a small VEV $v_{f} \gtrsim O(1-10) \mathrm{GeV}[15]$, or extended technicolor interactions [16].

We thank B. A. Dobrescu, J. Erler, A. Grant, C. T. Hill, and M. E. Peskin for valuable discussions, and G. Cvetic and E. H. Simmons for helpful comments on the manuscript. This work is supported by the NSF (PHY-9802564) and DOE (HEP Division, Contract W-31-109-Eng-38).
[1] For reviews, C.T. Hill, hep-ph/9702320; hep-ph/9802216; G. Cvetic, Rev. Mod. Phys. 71, 513 (1999).

[2] X. Li and E. Ma, Phys. Rev. Lett. 47, 1788 (1981).

[3] R.S. Chivukula, E.H. Simmons, and J. Terning, Phys. Lett. B 331, 383 (1994).

[4] E. Malkawi, T. Tait, and C.-P. Yuan, Phys. Lett. B 385, 304 (1996); D. Muller and S. Nandi, ibid. 383, 345 (1996).

[5] B.A. Dobrescu and C.T. Hill, Phys. Rev. Lett. 81, 2634 (1998); R.S. Chivukula et al., Phys. Rev. D 59, 075003 (1999).

[6] H. Collins, A. Grant, and H. Georgi, Phys. Rev. D 61, 055002 (2000)

[7] J. Erler and P. Langacker, Phys. Rev. Lett. 84, 212 (2000).
[8] M.E. Peskin and T. Takeuchi, Phys. Rev. D 46, 381 (1992).

[9] N. Maekawa, Phys. Rev. D 52, 1684 (1995).

[10] J. Erler and P. Langacker, review at http://pdg.lbl.gov

[11] H.E. Haber and H.E. Logan, Phys. Rev. D (to be published), hep-ph/9909335; M.L. Swartz, hep-ex/9912026.

[12] H.-J. He and C.-P. Yuan, Phys. Rev. Lett. 83, 28 (1999).

[13] W.-S. Hou et al., Phys. Lett. B 409, 344 (1997).

[14] H.-J. He, C.T. Hill, T. Tait, and C.-P. Yuan (in preparation).

[15] B.A. Dobrescu and E.H. Simmons, Phys. Rev. D 59, 015014 (1999).

[16] For recent reviews, K. Lane, hep-ph/9501249; R.S. Chivukula, hep-ph/9701322; hep-ph/9803219. 